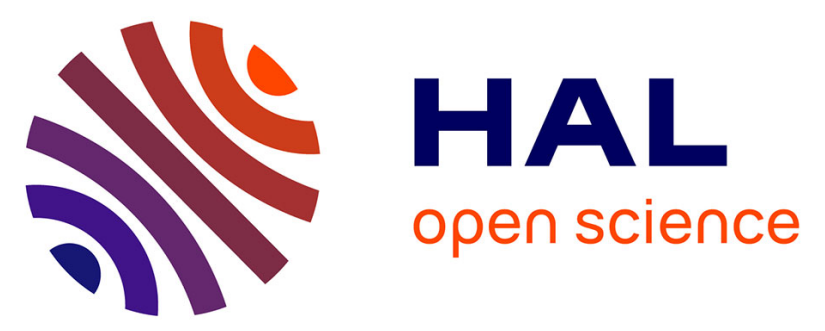

\title{
The Magnetar XTE J1810-197: Variations in Torque, Radio Flux Density, and Pulse Profile Morphology
}

F. Camilo, Ismaël Cognard, S.M. Ransom, J.P. Halpern, J. Reynolds, N. Zimmerman, E.V. Gotthelf, D.J. Helfand, P. Demorest, Gilles Theureau, et al.

\section{- To cite this version:}

F. Camilo, Ismaël Cognard, S.M. Ransom, J.P. Halpern, J. Reynolds, et al.. The Magnetar XTE J1810-197: Variations in Torque, Radio Flux Density, and Pulse Profile Morphology. The Astrophysical Journal, 2007, 663 (1), pp.497-504. 10.1086/518226 . insu-02778358

\section{HAL Id: insu-02778358 \\ https://hal-insu.archives-ouvertes.fr/insu-02778358}

Submitted on 4 Jun 2020

HAL is a multi-disciplinary open access archive for the deposit and dissemination of scientific research documents, whether they are published or not. The documents may come from teaching and research institutions in France or abroad, or from public or private research centers.
L'archive ouverte pluridisciplinaire HAL, est destinée au dépôt et à la diffusion de documents scientifiques de niveau recherche, publiés ou non, émanant des établissements d'enseignement et de recherche français ou étrangers, des laboratoires publics ou privés. 


\title{
THE MAGNETAR XTE J1810-197: VARIATIONS IN TORQUE, RADIO FLUX DENSITY, AND PULSE PROFILE MORPHOLOGY
}

\author{
F. Camilo, ${ }^{1}$ I. Cognard, ${ }^{2}$ S. M. Ransom, ${ }^{3}$ J. P. Halpern, ${ }^{1}$ J. Reynolds, ${ }^{4}$ N. Zimmerman, ${ }^{1}$ \\ E. V. Gotthelf, ${ }^{1}$ D. J. Helfand ${ }^{1}$ P. Demorest,${ }^{5}$ G. Theureau, ${ }^{2}$ and D. C. Backer ${ }^{5}$ \\ Received 2006 October 22; accepted 2007 March 19
}

\begin{abstract}
We report on 9 months of observations of the radio-emitting anomalous X-ray pulsar XTE J1810-197 starting in 2006 May using the Nançay, Parkes, Green Bank Telescope, and VLA telescopes mainly at a frequency of $1.4 \mathrm{GHz}$. The torque experienced by the neutron star during this period, as inferred from a measurement of its rotational frequency derivative, decreased by $60 \%$, although not in a steady manner. We have also observed very large ongoing fluctuations in flux density and pulse shape. Superimposed on these, a general diminution of flux density and a broadening of the pulse profile components occurred nearly contemporaneously with a decrease in torque of about $10 \%$ that took place in late 2006 July over an interval of 2 weeks. After a slight increase in average flux density, since 2006 October the flux density has continued to decline and the pulse profiles, while still varying, appear more uniform. In addition, a simultaneous observation of the pulsar with the Chandra X-ray Observatory and the Green Bank Telescope allows us to show how the X-ray and radio profiles are aligned. We discuss briefly the implications of these results for the magnetospheric currents in this remarkable object.
\end{abstract}

Subject headings: pulsars: individual (XTE J1810-197) — stars: neutron

\section{INTRODUCTION}

The transient anomalous X-ray pulsar (AXP) XTE J1810$197(P=5.54 \mathrm{~s})$ was discovered in early 2003 , when its X-ray luminosity increased $\sim 100$-fold (Ibrahim et al. 2004) compared to the quiescent state maintained for $>24 \mathrm{yr}$ (Halpern \& Gotthelf 2005). Initial X-ray observations revealed unsteady spin-down with $\dot{P} \approx 10^{-11}$ (implying a surface magnetic dipole field strength $B \approx 2 \times 10^{14} \mathrm{G}$ ) that varied by up to a factor of 2 within a few months of the outburst. Such rotational instability has been observed in other magnetars (see Woods \& Thompson 2006 for a review of the properties of magnetars).

The origin of a radio source positionally coincident with the pulsar (Halpern et al. 2005) was clarified by the detection of strong, narrow, highly linearly polarized radio pulses once per stellar rotation (Camilo et al. 2006). This emission has several unique characteristics. First, it is at least 1 order of magnitude brighter than in the late 1990s, when it was undetected; presumably the radio source turned on following the X-ray outburst. Just as the $\mathrm{X}$-ray flux has now decayed to a level approximating the historical low state (Gotthelf \& Halpern 2007), the radio emission can be expected to cease "shortly." In the initial observations, Camilo et al. (2006) also noted that the radio flux density of XTE $\mathrm{J} 1810-197$ varied on timescales of approximately 1 day in a manner inconsistent with interstellar scintillation, implying large day-to-day radio luminosity changes not seen in ordinary pulsars. The "average" pulse profiles were also found to change in a manner seemingly inconsistent with variations observed in other neutron stars. Lastly, the magnetar has a very flat radio spectrum

\footnotetext{
${ }^{1}$ Columbia Astrophysics Laboratory, Columbia University, New York, NY 10027.

${ }^{2}$ Laboratoire de Physique et Chimie de l'Environnement, CNRS, F-45071 Orleans, Cedex 2, France.

${ }_{3}^{3}$ National Radio Astronomy Observatory, Charlottesville, VA 22903.

4 Australia Telescope National Facility, CSIRO, Parkes Observatory, Parkes, NSW 2870, Australia.

5 Department of Astronomy, University of California, Berkeley, CA 947203411.
}

and has been detected at higher radio frequencies than any other pulsar.

These unusual characteristics of XTE J1810-197 presumably reflect different physical conditions in its magnetosphere compared with those of both ordinary radio pulsars and persistent magnetars, which so far have shown no evidence of magnetospheric radio activity (e.g., Burgay et al. 2006). The study of this radio emission thus provides a new window into the coronae of magnetars. We summarize here some results from our monitoring program of XTE J1810-197, focusing on rotational, flux density, and pulse profile evolution.

\section{DATA ACQUISITION, ANALYSIS, AND RESULTS}

\subsection{Observations}

\subsubsection{Nançay}

We have observed XTE J1810-197 at Nançay since 2006 June 1 (MJD 53887) with typical integration times of 15-60 minutes using the Berkeley-Orleans-Nançay (BON) coherent dedispersor (Cognard \& Theureau 2006) based on a Serendip V spectrometer. ${ }^{6}$ The Nançay radio telescope has a gain of $1.5 \mathrm{~K} \mathrm{Jy}^{-1}$ and a system temperature of $47 \mathrm{~K}$ at $1.4 \mathrm{GHz}$ in the direction of XTE J1810-197, which this meridian-type telescope can track for $1 \mathrm{hr}$ each day. We monitored the pulsar nearly every day until 2006 October, and have done so every $2-3$ days on average since then. The last observations reported on here are from 2007 January 27. Dedispersion of a $64 \mathrm{MHz}$ band centered on $1398 \mathrm{MHz}$ is done coherently into sixteen $4 \mathrm{MHz}$ channels using a 64-node computer cluster, with each data stream then folded every 2 minutes at the pulsar's predicted period. In Figure 1 we show the daily folded pulse profiles obtained in the linear horizontal polarization (with dipole orientation parallel to the ground) after excising significant levels of radio frequency interference (RFI). Regional telephone relay and radio transmitters use the

\footnotetext{
${ }^{6}$ See http://seti.berkeley.edu/casper/projects/SERENDIP5/.
} 


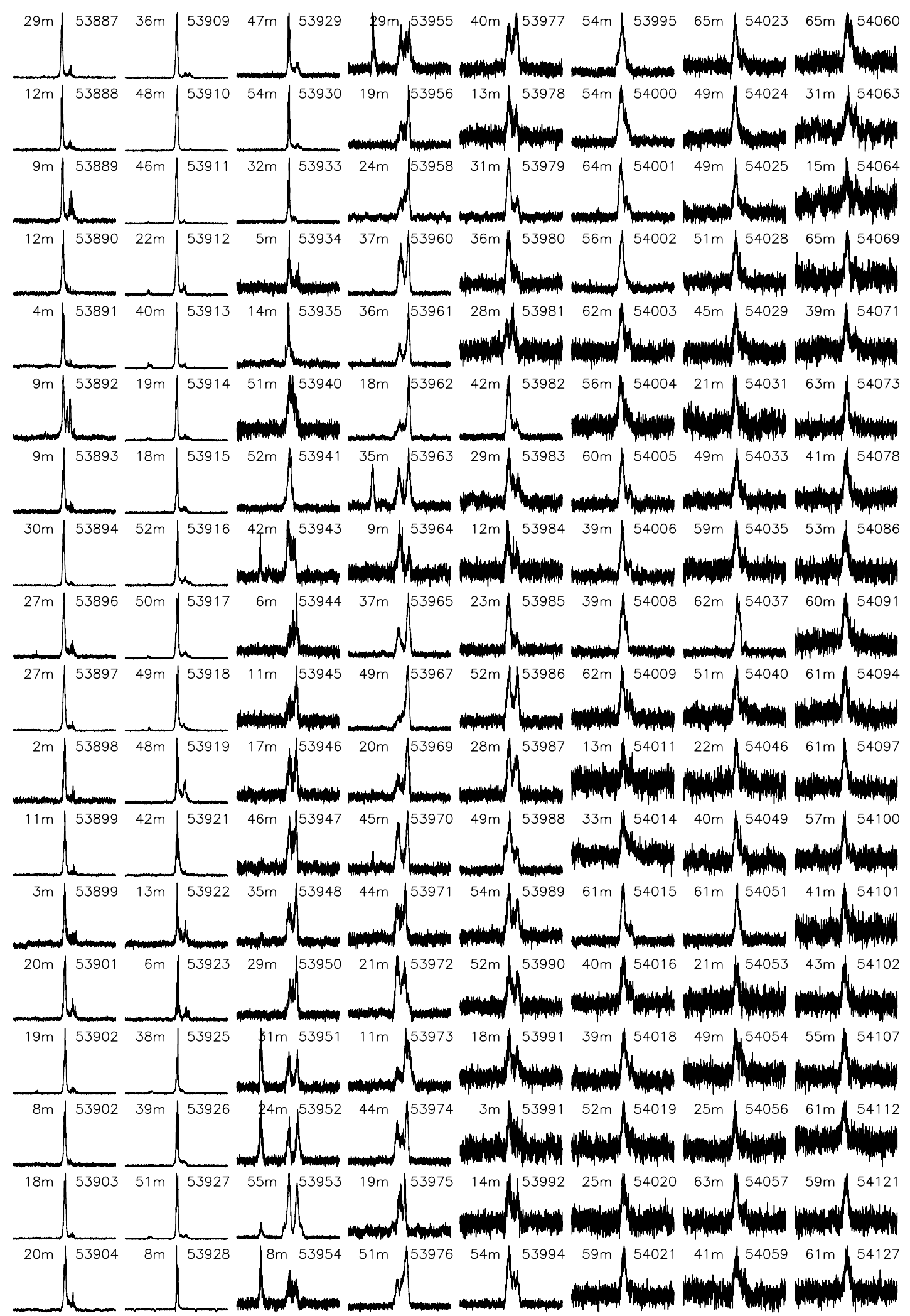

FIG. 1.-Daily pulse profiles from XTE J1810-197 recorded in the linear horizontal polarization at Nançay at a frequency of $1.4 \mathrm{GHz}$ across a bandwidth of $64 \mathrm{MHz}$ (§ 2.1.1), each displayed with 2048 bins (the first is from 2006 June 1 and the last from 2007 January 27). We aligned these profiles using an ephemeris containing 11 frequency derivatives that resulted in featureless residuals (see $\S 2.2$ ). Each profile is labeled with the effective integration time (i.e., after the removal of RFI) in minutes to its left and the observing date (MJD) to its right. In some cases minor artifacts remain, due to incompletely excised RFI. 
vertical polarization, leading to much more severe RFI in that channel. However, the radio emission from XTE J1810-197 is highly polarized (Camilo et al. 2007) and fortuitously is much stronger in the direction parallel to the ground at Nançay, so that the vertical signal is in any case usually very small (the parallactic angle change during the maximum integration is a negligible $\left.\pm 2.5^{\circ}\right)$.

Since 2006 July we have used a second observing system in parallel with BON, where square-law detectors sampled every $2 \mathrm{~ms}$ record the total power for each polarization channel in a $50 \mathrm{MHz}$ band centered on $1410 \mathrm{MHz}$. These data are flux calibrated by the firing of a calibrated pulsed noise diode at the beginning of each observation. However, this detector's sampling rate is not stable enough for pulsar timing purposes, for which we use the accurately time-tagged $\mathrm{BON}$ records.

We have used data from both systems to obtain flux density estimates for the daily profiles. The square-law detected outputs suffer less from RFI (because of their higher central frequency and narrower bandwidth) and are accurately flux calibrated. On the other hand, removal of RFI in these records is complicated by the lack of frequency resolution. For BON data we first carefully removed RFI in the time-frequency plane and then used the radiometer equation, along with the known telescope noise characteristics, to convert the rms noise fluctuation in the off-pulse part of the profiles to a Jansky scale. We determined this conversion factor for the horizontal polarization and applied it to both channels. We then added the corresponding profiles from both polarizations and, by integrating the area under each profile, finally measured the pulsed flux density. On most days the flux densities estimated from the two systems agree to within $\sim 25 \%$, and hereafter we use only the BON values.

It is evident from Figure 1 that the pulse profiles are not stable, particularly for the 2 months following late 2006 July (MJD 53935 is July 19). This presents obvious difficulties for obtaining pulse times of arrival (TOAs) by following the usual prescription of cross-correlating a "standard profile" with each day's profile. Instead, we obtain TOAs from the maximum of a parabola fitted to one pulse profile component that we assume, regardless of variations, corresponds to the same longitudinal fiducial point on the pulsar. We choose for this fiducial point the left-most of the (typically) two components that make up the main cluster of emission. This choice is motivated by the facts that, within our large and densely sampled set of observations, (1) the longitudinal separation between the corresponding peaks $(0.08 P)$ remains essentially fixed even as the profiles vary enormously (e.g., see the profiles for MJDs 53929 and 53977 in Fig. 1, for which the fiducial points are effectively the peaks of, respectively, the highest and lowest clearly visible pulse components); and (2) the angular separation between the left-most peak and the "precursor" visible on some days, 0.3 in pulse phase preceding it (which is generally much fainter than the main components, but not always: see, e.g., the profiles for MJDs 53925 and 53951), also remains constant within the uncertainties. Also commending this method is the empirical consideration that it works well, with only a handful of TOAs rejected, because their prefit residuals are particularly large, owing presumably to "awkward" pulse shapes. The TOAs thus obtained for timing purposes $(\S 2.2)$ also match (up to a constant offset of $\approx 25 \mathrm{~ms}$ ) those obtained via the standard cross-correlation method for data acquired before mid 2006 July (when profile variation was relatively small).

\subsubsection{Parkes}

The first detection of radio pulsations from XTE J1810-197 took place at the ATNF Parkes telescope on 2006 March 17, with confirmation following on April 25 (Camilo et al. 2006). Since then we have observed the pulsar there at frequencies ranging from 0.7 to $8.4 \mathrm{GHz}$ using a variety of spectrometers. Unless otherwise noted, in this paper we use only $1.4 \mathrm{GHz}$ data, obtained with the analog filterbanks as described by Camilo et al. (2006).

The $1.4 \mathrm{GHz}$ Parkes data set is very sparse by comparison with Nançay's, but is particularly useful here for two purposes: to extend the overall timing span by more than 1 month, and to perform consistency checks on the timing solution using two independent sets of TOAs. Because the vast majority of the Parkes data used here were obtained before late 2006 July (when pulse shapes were relatively stable; see Fig. 1 and $\S 2.1 .1$ ), the standard cross-correlation method that we used to obtain these TOAs works well enough.

\subsection{3. $G B T$}

We have observed XTE J1810-197 at the NRAO Green Bank Telescope (GBT) since 2006 May 2 at frequencies spanning $0.3-42 \mathrm{GHz}$. Very few of these data are at $1.4 \mathrm{GHz}$. Starting in 2007 January, after the monitoring rate decreased substantially at Nançay (§ 2.1.1), we increased GBT monitoring at $1.9 \mathrm{GHz}$, with data collected as described by Camilo et al. (2006). The TOAs derived from these data, using the standard cross-correlation method with pulse profiles that vary little, are used to complement Nançay TOAs. Also, using the large gain, bandwidth, and frequency agility of GBT, we have obtained very high quality pulse profiles at a number of widely separated frequencies that illustrate the remarkable time variation in pulse shapes displayed by this magnetar. Some examples of this collection at a frequency of $1.9 \mathrm{GHz}$ are shown in Figure 2.

\subsubsection{VLA}

We have used a subarray of the NRAO Very Large Array (VLA) to measure with high precision the flux density of XTE J1810-197 at $1.4 \mathrm{GHz}$ on 13 days between 2006 February 28 and September 5. During a typical observation we accumulate 40 minutes of on-source visibilities in continuum mode, with a bandwidth of $100 \mathrm{MHz}$. Each observation begins with $\mathrm{a} \approx 5 \mathrm{~min}-$ ute scan of a flux calibrator, either 3C 286 or $3 \mathrm{C} 48$. The remainder of the observation consists of three $\approx 2$ minute scans of the phase calibrator $1811-209$ interspersed with two $\approx 20$ minute on-source scans. The recorded visibilities are calibrated and imaged using standard procedures in the Astronomical Image Processing System. Due to variations in the array configuration, the number of antennas used, and the amount of observing time allocated, the errors in our flux density measurements range between 0.2 and $1.0 \mathrm{mJy}$.

\subsubsection{Chandra}

XTE J1810-197 was observed with the Chandra X-ray Observatory on 2006 September 10-11 using the back-illuminated S3 chip of the ACIS-S CCD detector in TIMED/VFAINT mode with a subarray readout that provided a time resolution of $0.441 \mathrm{~s}$ and a dead time of $9 \%$. Data were acquired continuously over an interval of $8.4 \mathrm{hr}$ starting at 19:40 UT with no background contamination. All photon arrival times were corrected to the solar system barycenter (TDB) using the pulsar position given in Table 1, the JPL DE200 ephemeris, and the axbary task in the Chandra Interactive Analysis of Observations. Photons falling within an aperture of radius 3 " centered on XTE J1810-197 were extracted from the standard processing event level 2 data files, and a total of 7014 counts were accumulated in the $0.5-3.0 \mathrm{keV}$ energy range, chosen to maximize the source signal-to-noise ratio. 


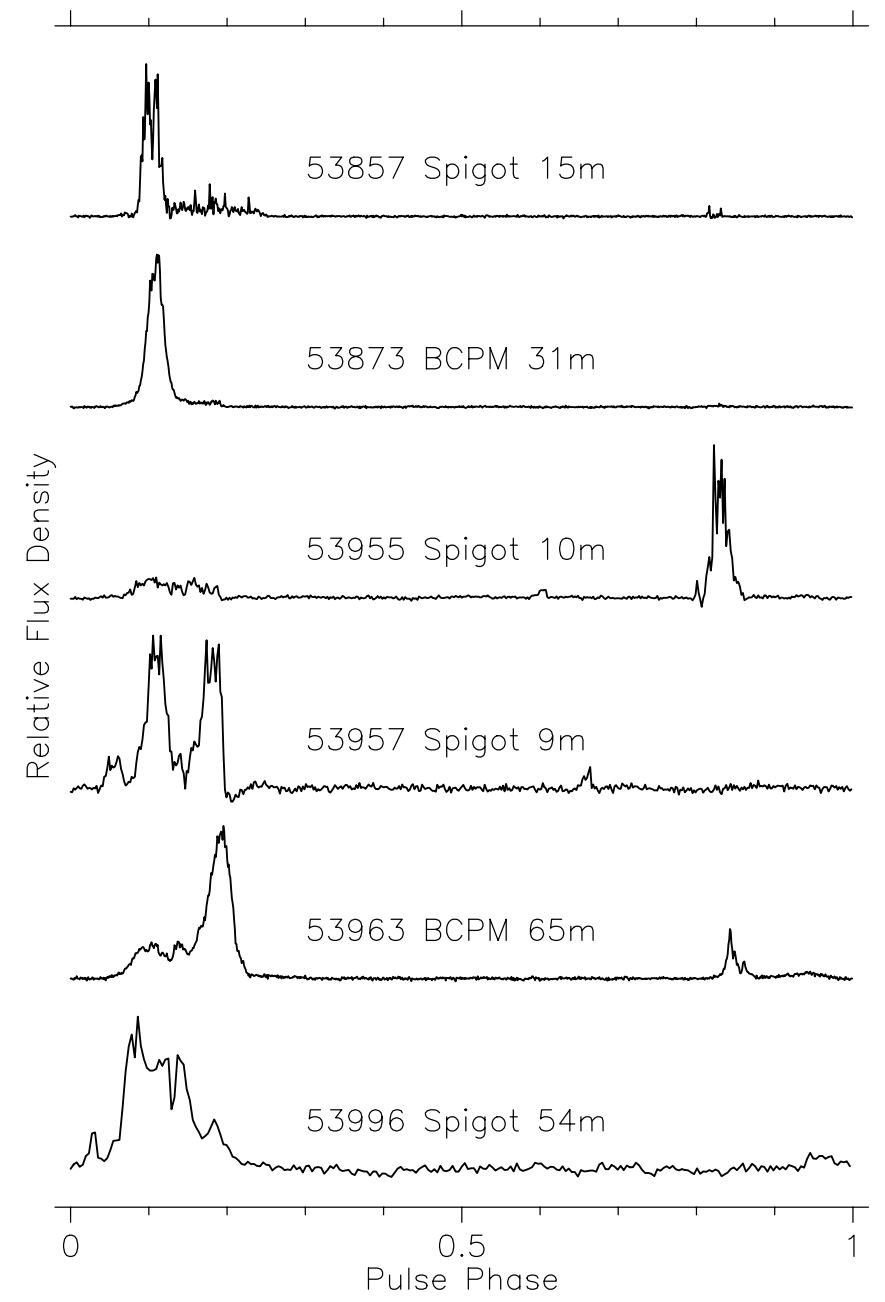

FIG. 2.-Changing pulse shapes of XTE J1810-197 from GBT observations. All profiles were aligned with the polynomial used for Fig. 1. The labels list the observation date (MJD), spectrometer used, and effective integration time in minutes. Spigot (Kaplan et al. 2005) data were recorded across a $600 \mathrm{MHz}$ band centered at $1.95 \mathrm{GHz}$, while a $134 \mathrm{MHz}$ band centered on $1.85 \mathrm{GHz}$ was used for BCPM (Backer et al. 1997). Note the low-level emission near phases 0.8 on MJD 53857, 0.6 on MJD 53955, 0.65 on MJD 53957, and 0.95 on MJD 53963. See also Fig. 5.

The barycentered photon arrival times were folded at epoch MJD 53989.0 (midnight TDB on September 11) into 10 bins using $P=5.540362 \mathrm{~s}$ determined from a simultaneous radio observation at the GBT (see $\S 2.2 .1)$.

\subsection{Timing}

We have used the available TOAs ( $\S \S 2.1 .1-2.1 .3)$, along with the $\mathrm{TEMPO}^{7}$ software, to obtain the timing solution that describes the rotational history of XTE J1810-197 during 2006 May-2007 January. Because of the changing pulse shapes and heterogeneity of the available data $(\S 2.1)$, particular care must be taken with this process.

First we used Nançay TOAs to obtain a solution beginning in 2006 June. Starting with a handful of TOAs and an initial solution fitting only for rotational phase and frequency $\nu=1 / P$, we subsequently added $\dot{\nu}$ as needed. We then added one TOA at a time, paying particular attention to its prefit residual and corresponding pulse shape, as well as the postfit residuals and fit parameters. In this way we eliminated seven TOAs, from a set of

\footnotetext{
${ }^{7}$ See http://www.atnf.csiro.au/research/pulsar/tempo/.
}

TABLE 1

Timing Parameters for XTE J1810-197

\begin{tabular}{|c|c|}
\hline Parameter & Value \\
\hline R.A. & $18^{\mathrm{h}} 09^{\mathrm{m}} 51.087^{\mathrm{s}}$ \\
\hline 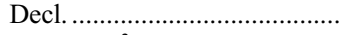 & $-19^{\circ} 43^{\prime} 51.93^{\prime \prime}$ \\
\hline $\mathrm{DM}\left(\mathrm{cm}^{-3} \mathrm{pc}\right) \ldots \ldots$ & 178.0 \\
\hline 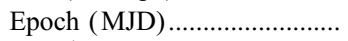 & 54000.0 \\
\hline$\nu\left(\mathrm{s}^{-1}\right)^{\mathrm{a}} \ldots \ldots$ & $0.180493891(6)$ \\
\hline$\dot{\nu}\left(10^{-13} \mathrm{~s}^{-2}\right)^{\mathrm{a}} \ldots \ldots \ldots \ldots \ldots \ldots$ & $-2.53(1)$ \\
\hline MJD range & $53850-54127$ \\
\hline
\end{tabular}

Notes.-The celestial coordinates were held fixed at the values obtained from VLBA observations (Helfand et al. 2007), and the DM was held fixed at the value obtained from simultaneous 0.7 and $2.9 \mathrm{GHz}$ observations (Camilo et al. 2006).

${ }^{a}$ These two parameters are sufficient to obtain a phase-connected solution encompassing the MJD range, but do not fully describe the rotation of the neutron star. They are nonstationary and, strictly, not predictive. See Fig. 3 and $\S 2.2$ for more details.

144 , that had large ( $\gtrsim 100 \mathrm{~ms}$ ) prefit residuals; these could usually be ascribed to particularly unusual pulse shapes. After approximately 1 month it is no longer possible to obtain featureless timing residuals without fitting for higher frequency derivatives. After this we added the Parkes TOAs, fitting also for an offset between both TOA sets to account for an arbitrary alignment between the respective fiducial points. The magnitude of this offset $(21 \pm 15 \mathrm{~ms}$; unless otherwise stated, the uncertainties used in this paper all represent $1 \sigma$ confidence levels) is as expected, considering the different methods used to obtain the respective TOAs (see $\S \S 2.1 .1$ and 2.1.2). Finally, when measuring $\dot{\nu}$ in piecewise fashion for 2007 January, we complemented the Nançay TOAs with those from the GBT $(\S 2.1 .3)$.

In general it is difficult to estimate TOA uncertainties reliably for our data, owing to changing pulse shapes and the principal method used to obtain TOAs (see $\S 2.1 .1$ ). For the early Parkes data these are typically in the $5-10 \mathrm{~ms}$ range. The uncertainty of early Nançay TOAs is more often 10-20 ms. Following the general weakening of the pulsar, the broadening of its profile components, and the huge variability displayed by the pulse shapes (Fig. 1), we estimate that the TOA uncertainties are commonly around $25-50 \mathrm{~ms}$ but can reach values approaching $100 \mathrm{~ms}$ on occasion. As noted above we have excluded from the timing fits a few TOAs with such large prefit residuals (and estimated uncertainties). Because of these difficulties, we assigned a uniform weight to the TOAs, and as a result, we do not obtain a $\chi^{2}$ figure of merit for the timing fits. Nevertheless, the TOA uncertainty estimates noted here are in keeping with the corresponding fit rms residuals.

We obtained a phase-connected timing solution that encompasses all data beginning with the confirmation TOAs from April 25 (we comment below on use of the discovery TOAs from 2006 March 17). The residuals from a quadratic fit to pulse phase are shown in the top panel of Figure 3, and the respective solution is given in Table 1. An independent solution using only Parkes TOAs results in similar parameters. The cubic trend of large amplitude visible in the residuals points to very significant unmodeled behavior, expected of young pulsars in general (whose rotation is "noisy") and of XTE J1810-197 in particular (Ibrahim et al. 2004). While $\dot{\nu}$ clearly varies with time, the bottom panel of Figure 3 shows that it does not do so smoothly. A total of 11 frequency derivatives are required to "whiten" the residuals, which then have an rms of $18 \mathrm{~ms}$. 


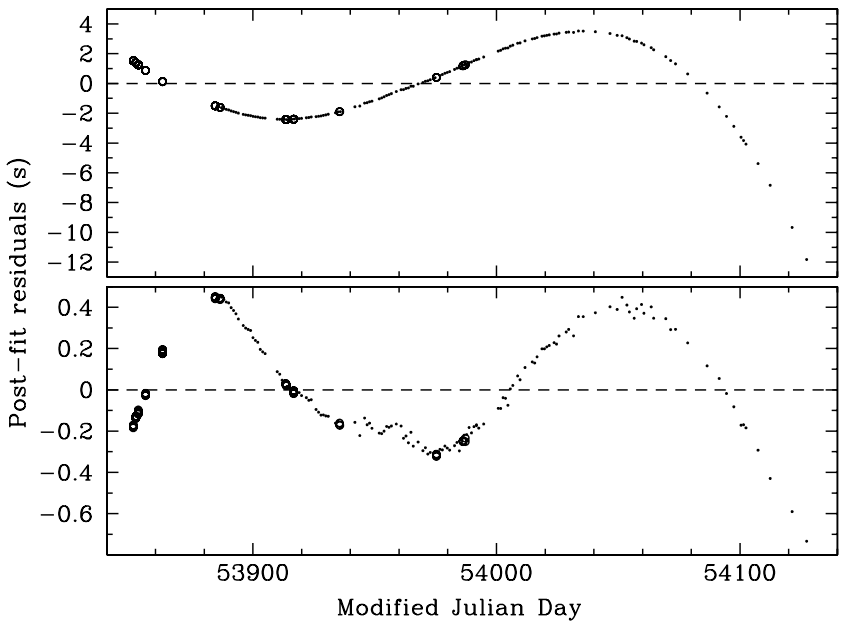

FIG. 3.-Timing residuals for XTE J1810-197. Black dots correspond to Nançay TOAs, while open circles represent Parkes TOAs (see § 2.1). Top: Residuals for a model that contains only the rotation phase, frequency $\nu$ and $\dot{\nu}$ (see Table 1), showing a clear cubic residual trend. Bottom: Residuals for a model with an additional polynomial term, $\ddot{\nu}=9.40 \pm 0.06 \times 10^{-21} \mathrm{~s}^{-3}$. Note the change in vertical scale by a factor of about 10 , and the remaining quartic trend.

In order to extract from this record of unsteady rotation a quantitative measure of the varying torque acting on the neutron star, we have measured $\dot{\nu}$ as a function of time. We have done this in a piecewise fashion, performing quadratic fits to phase for TOA spans long enough that the nominal resulting fractional uncertainty in $\dot{\nu}$ was no more than $3 \%$, but short enough that no trends were seen in the residuals. In practice this implied individual segments of very nearly 30 days each. So as to better sample the variation in $\dot{\nu}$, we did this with Nançay data (supplemented in 2007 January by GBT TOAs) while stepping through the TOAs in offsets of 15 days. A fit to all the good Nançay TOAs using 11 frequency derivatives also yields $\dot{\nu}(t)$ (without associated uncertainties) consistent with the discrete measurements.

Owing to the lack of Nançay observations and the sparser data from Parkes and GBT, this was not straightforward before 2006 June 1 . We opted for performing timing fits using multifrequency data from both Parkes and GBT, thereby gaining some additional leverage in our solutions. At this time, the radio emission at most frequencies was usually dominated by a single narrow component (see Camilo et al. 2006), so that TOA artifacts were relatively small. In this manner, we obtained a $\dot{\nu}$ measurement for late April-late May. Finally, we also used the 2006 March 17 Parkes discovery observations in a timing fit. We appear to have maintained phase connection with the confirmation observations 39 days later (the prefit residual between the discovery TOAs and the solution computed with the first month of data following confirmation is only $0.16 P$ ). However, we have established with Nançay data that cubic trends typically become significant for $\gtrsim 30$ days, and we have therefore obtained this earliest measurement of $\dot{\nu}$ also by computing the difference in barycentric frequencies between 2006 March 17 and April 25. The two $\dot{\nu}$ values differ by only $2.7 \sigma$ of the (much smaller) uncertainty of the phase-connected fit, and this agreement allows us to extend our record of $\dot{\nu}$ by 1 additional month.

The run of $\dot{\nu}$ measured over 9 months is shown in the top panel of Figure 4 . The overall trend is one of increasing $\dot{\nu}$ (as could be inferred from the top panel of Fig. 3), but the way in which this occurs is far from steady. In the 3 months prior to mid 2006 July, $\dot{\nu}$ varied in a relatively steady and gradual fashion from $-3.3 \times 10^{-13} \mathrm{~s}^{-2}$ to $-3.0 \times 10^{-13} \mathrm{~s}^{-2}$. Then, in late July, it

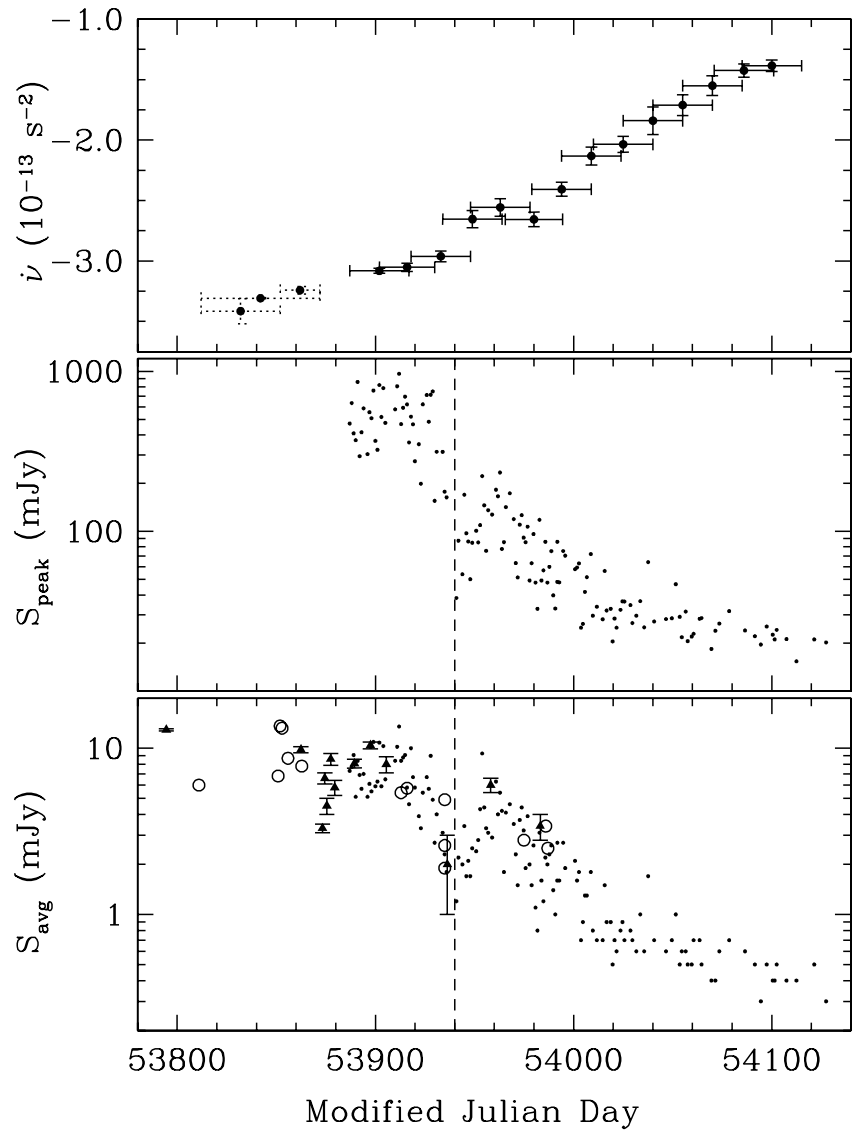

FIG. 4.-Frequency derivative and flux densities at $1.4 \mathrm{GHz}$ for XTE J1810197. Top: Frequency derivative obtained from a variety of timing fits each spanning about 30 days. Solid error bars denote fits obtained with Nançay data alone (excepting the last one, which also contains some GBT data), while dotted error bars correspond to fits that contain Parkes and in some cases also GBT data. For fits containing Nançay data the error bars represent nominal $2 \sigma$ confidence levels; for all others, $4 \sigma$. In late 2006 July (indicated by the dashed line at MJD 53940 in the lower panels), $\dot{\nu}$ increased at a large rate, the flux densities reached a local minimum, and the pulse profiles changed in character. See $\S 2.2$ for details. Middle: Daily peak flux density of the profile component used to obtain Nançay TOAs (see $\S 2.1 .1$ and Fig. 1). Bottom: Daily period-averaged flux density from Nançay (black dots) and Parkes (open circles), and continuum VLA flux density (triangles with error bars).

changed to $-2.7 \times 10^{-13} \mathrm{~s}^{-2}$ over a span of 15 days; the implied change in torque is $-2 \times 10^{32} \mathrm{dyn} \mathrm{cm}$ (using a stellar moment of inertia of $10^{45} \mathrm{~g} \mathrm{~cm}^{2}$ ), which is larger than the torque powering three-quarters of all known ordinary pulsars! Regardless of the detailed mechanisms that produce radio pulses in XTE J1810-197, it should perhaps not be surprising if other observational properties of the magnetar should have changed around this time.

In fact, as the middle panel of Figure 4 shows, the peak flux density of XTE J1810-197 has dramatically decreased since about that time. Interestingly, while the period-averaged flux density has also decreased compared to its average value before mid July (bottom panel of Fig. 4), until about 2006 October it did so by a smaller factor and continued to fluctuate greatly from day to day (when average flux densities are available from at least two of Nançay, Parkes or VLA within 1 day of each other, they are consistent within expectations given the inherent variations). These two observations can be understood by inspection of the profiles shown in Figure 1: from late July to mid September (MJD 53994), the daily profiles of XTE J1810-197 tended to be composed of two (or more) significant peaks, each much broader than the one peak generally prominent before mid July 


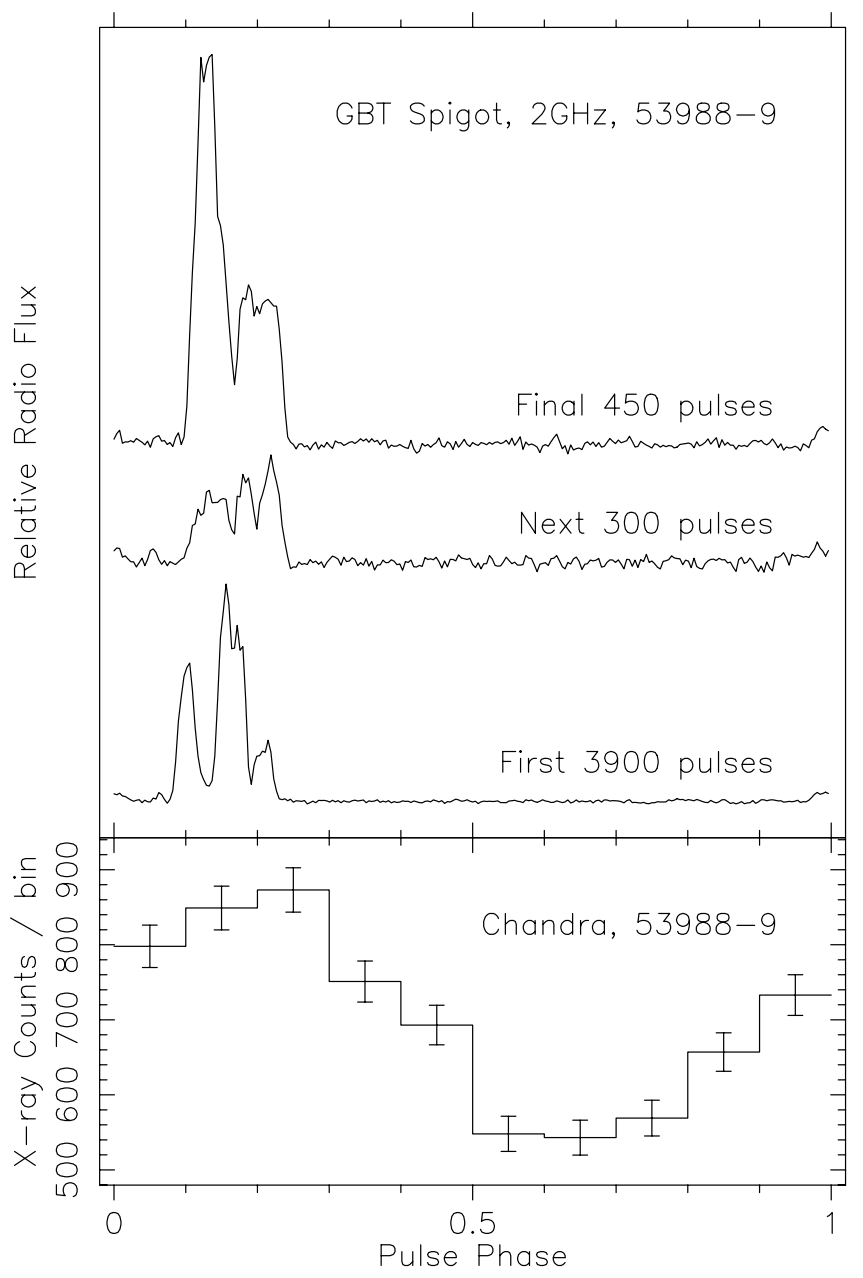

FIG. 5.-Bottom: X-ray pulse profile $(0.5-3.0 \mathrm{keV})$ of XTE J1810-197 from an observation with Chandra on 2006 September 10-11, folded with barycentric $P=5.540362 \mathrm{~s}$ as determined from a simultaneous radio observation (see $\S 2.1 .5$ ). Phase zero on this plot corresponds to MJD 53989.0 (TDB). Top: Radio profiles from a simultaneous GBT observation, starting on September 10, 20:50 UT. After $6 \mathrm{hr}$ (bottom trace), the pulse profile abruptly changed to a different configuration for 30 minutes (middle trace), after which it changed yet again (top trace). The relative integrated flux densities of the three profiles are 1.4, $1.0,2.9$, from bottom to top. The middle peak of the bottom radio profile arrives at phase 0.17 on the plot, and all four profiles are absolutely aligned (see $\S 2.2 .1$ ). Note also the alignment between the left-most peak of the top profile and the first notch in the bottom profile.

(with typical full width at half-maximum $\approx 0.04 P$, versus about half of that value beforehand), and with greater pulse-shape variance than before. This may also explain why the Nançay timing residuals for each 1-month fit after mid 2006 July are about $24 \mathrm{~ms}$ rms, twice as large as the typical corresponding value before then.

Since 2006 October, the Nançay profiles appear to have varied less and to be composed mainly of one broad peak (Fig. 1), although on some days the trailing peak is still recognizable (as it is more often in higher quality GBT data). After another relatively large increase over the month of September, $\dot{\nu}$ has continued a steady increase at approximately the average rate for the 9-month span of our observations, $7.5 \times 10^{-16} \mathrm{~s}^{-2}$ per day.

\subsubsection{X-Ray and Radio Pulse Alignment}

On 2006 September 10-11 we observed XTE J1810-197 for $7.2 \mathrm{hr}$ with the GBT at $1.9 \mathrm{GHz}$, starting $1 \mathrm{hr}$ after the beginning of the Chandra observation ( $\S 2.1 .5)$. We have used the contemporaneous TOAs thus obtained to measure the phase offset between the X-ray and radio pulses. The folded X-ray profile is shown in Figure 5, with the first phase bin chosen to begin at midnight on September 11 (TDB). After translating the TOAs for the first radio profile (see Fig. 5) converted to infinite frequency to the solar system barycenter, a TEMPO fit yields a phase offset for the fiducial point of the radio profile (here its middle peak) of $0.167 \pm 0.006$ in Figure 5. The uncertainty includes a component due to the fit and a slightly smaller contribution from the uncertainty in the dispersion measure (DM) of XTE J1810-197.

Within the larger uncertainty imposed by the Chandra resolution $(0.08 P$; see $\S 2.1 .5)$ and the relatively small number of pulsed X-ray counts, the main component of the radio profile on this day arrives at the same time as the peak of the X-ray pulse (Fig. 5).

Interestingly, the radio profile changed in both shape and flux during the Chandra observation, twice within a span of $30 \mathrm{~min}-$ utes (Fig. 5). This does not modify the conclusion in the above paragraph, but helps answer a question concerning the radio flux and profile variations observed in XTE J1810-197: they can occur suddenly (observed at a resolution of $\sim 10 \mathrm{~s}$ ). We have "caught in the act" at least nine such changes at Nançay, GBT, and Parkes, all since mid 2006 July. As this corresponds to some $150 \mathrm{hr}$ of observing time, we can estimate that such transitions occur on average every $\sim 15 \mathrm{hr}$ at the present epoch. No X-ray bursts or significant changes in the X-ray flux or pulse shape were seen at the times of the radio transitions indicated in Figure 5. The Chandra count rates during the three radio pulse states $-0.235 \pm 0.003,0.213 \pm 0.011$, and $0.218 \pm 0.007 \mathrm{~s}^{-1}$, respectively - are uncorrelated with the large changes in radio flux density and pulse shape.

\section{DISCUSSION}

All young radio pulsars experience rotational instabilities, observed as a continuous quasi-stochastic wandering of the pulse phase ("timing noise") or as discontinuous spin-up events ("glitches"). These are thought ultimately to be driven by the unsteady transfer of angular momentum from the interior superfluid to the crust of the neutron star. XTE J1810-197 displays large amounts of timing noise-like behavior; despite very large changes observed in $\dot{\nu}$ on short timescales, all observed rotational changes have been continuous to within the available resolution, and we have not observed any clear glitch-like behavior. Arzoumanian et al. (1994) quantified timing noise as the time magnitude of its cumulative contribution over a time interval $t$ to the cubic term in a Taylor series expansion of rotational phase, i.e., $\ddot{\nu} t^{3} / 6 \nu$. For XTE J1810-197 this amounts to $120 \mathrm{~s}$ over 277 days, a huge amount that far surpasses anything observed in radio pulsars and that is also greater than an extrapolation based on the notion that the magnitude of timing noise is proportional to $\dot{P}$ (Arzoumanian et al. 1994). However, this level of timing noise is not unprecedented for magnetars.

Six AXPs, including XTE J1810-197, and two soft-gamma repeaters (SGRs) - i.e., the majority of all known magnetars have been observed with phase connection maintained for at least a few months (see Kaspi et al. 2001; Gotthelf et al. 2002; Gavriil \& Kaspi 2002, 2004; Woods et al. 2002; Kaspi \& Gavriil 2003). The SGRs $1806-20$ and $1900+14$ usually have the largest amounts of timing noise, roughly 1 order of magnitude above the level we observe in XTE J1810-197, with $\dot{\nu}$ varying by factors of up to 4 (Woods et al. 2002). The rotational stability of AXPs is generally greater (although at least two have been observed to glitch; see Kaspi et al. 2003; Kaspi \& Gavriil 2003), and four are substantially quieter than XTE J1810-197. The 
remaining one, 1E 1048.1-5937, was relatively quiet during 2005-2006 (Kaspi 2007), but previously displayed far greater rotational instability than observed in XTE J1810-197 (Gavriil \& Kaspi 2004). Therefore, while noisier than most AXPs, XTE J1810-197 may not be particularly remarkable in this respect. What is unusual is that we can now track its rotation via nearly daily observations, in greater detail than is possible for other AXPs.

Following its discovery with the Rossi X-ray Timing Explorer, a phase-connected solution was obtained for XTE J1810-197 spanning 2003 January-September, during which $\dot{\nu}$ varied between $-6.7 \times 10^{-13} \mathrm{~s}^{-2}$, averaged over 8 months, and $-3.8 \times$ $10^{-13} \mathrm{~s}^{-2}$ over the last 2 months (Ibrahim et al. 2004). The very large initial magnitude may have reflected in part transients associated with a putative glitch at the time of the X-ray outburst in early 2003. In the succeeding 2.5 years, until the radio detection, phase connection was no longer possible owing to the sparse sampling, but individual period measurements 6 months apart established, e.g., that the average $\dot{\nu}$ was $-1.7 \times 10^{-13} \mathrm{~s}^{-2}$ between late 2003 and early 2004 (see Gotthelf \& Halpern 2005). The value of $\dot{\nu}=-1.4 \times 10^{-13} \mathrm{~s}^{-2}$ in early 2007 is the largest ever measured for this source, for which the historical range can be summarized as $\dot{\nu}=(-2.6 \pm 1.2) \times 10^{-13} \mathrm{~s}^{-2}$ spanning late 2003-early 2007. Importantly, although $\dot{\nu}$ has increased almost monotonically during our radio observations (Fig. 4), it cannot have been increasing during the entirety of the past 3 years (see also Gotthelf \& Halpern 2007). Periods of decreasing torque such as we have observed recently must have been interspersed with at least one epoch of substantially increasing torque. If the torque for XTE J1810-197 were to continue decreasing at the rate in 2007 January, by mid 2007 the pulsar would stop spinning down altogether; presumably this rate will soon decrease.

In ordinary pulsars, the observed rotational instabilities are mainly presumed to be caused by angular momentum transfer processes internal to the neutron star. In the magnetar model, the external energy stored in the magnetosphere, partly released through reconfiguration and decay of the magnetic field and currents, may also lead to erratic variations in the torque acting on the star, for example via a flux of Alfvén waves and particles resulting from magnetically induced (sudden large-scale or persistent small-scale) crustal seismic activity (e.g., Thompson et al. 2000). It is unclear whether any such particular models (see also Duncan 2001) can explain the magnitude and timescale of the variations in torque currently observed in XTE J1810-197, 4 years after the X-ray outburst. In any case, substantial variations in magnetospheric plasma densities and/or currents would likely have implications for other observable properties of the pulsar.

An interesting comparison is with PSR B1931+24, an ordinary radio pulsar during intervals of 5-10 days, with $P=0.8 \mathrm{~s}$ and $B \approx 2 \times 10^{12} \mathrm{G}$, which abruptly shuts off for $25-35$ days in a pattern that repeats quasi-periodically (Kramer et al. 2006). During these turned-off periods, the torque is only $2 / 3$ of its turned-on value. Kramer et al. (2006) conclude from this extraordinary behavior that the occasional presence of plasma leads to radio emission, and its flow provides the extra braking torque. The plasma density calculated from the torque difference is $100 \mathrm{esu} \mathrm{cm}^{-3}$, in agreement with the corotation value (Goldreich \& Julian 1969). In XTE J1810-197 the radio emission has not shut off, but it did diminish and change markedly in character somewhat abruptly in late 2006 July, apparently coincident with a huge reduction in torque $\left(\dot{\nu}\right.$ changed from $-2.96 \times 10^{-13} \mathrm{~s}^{-2}$ on MJD 53933 to $-2.65 \times 10^{-13} \mathrm{~s}^{-2}$ on MJD 53949; see $\S 2.2$ and Fig. 4). A calculation such as that by Kramer et al. (2006) would suggest for XTE J1810-197 an "extra” plasma density of about 300 esu $\mathrm{cm}^{-3}$ prior to the large torque decrease, compared to the source's Goldreich-Julian density of $1350 \mathrm{esu} \mathrm{cm}^{-3}$. Implicit in this calculation is the notion that the radio emission originates from open field lines, which has not been proven for XTE J1810-197. For a magnetar, there is additional plasma due to nonaxisymmetric large-scale magnetospheric currents (Thompson et al. 2002). In any case, the pulse profile and flux variations observed in XTE J1810-197 occur on timescales that are apparently too short to be explained by changes in the closed field lines (see, e.g., Beloborodov \& Thompson 2007). Also, interpretation of polarimetric data for XTE J1810-197 appears consistent with emission from open field lines (Camilo et al. 2007).

If indeed the measured changes in torque are caused by variations in the magnetospheric plasma density in locations relevant for the production of coherent radio emission, then it should be no surprise that the pulse profiles of XTE J1810-197 change so remarkably: even the "small" torque changes reflected in Figure 4 are enormous by the standards of ordinary pulsars. And certainly, the profile variations in XTE J1810-197 (Figs. 1, 2, and 5) are observationally distinct from the "mode changing" of some ordinary pulsars, where the average pulse profile suddenly changes between two of a small set of different configurations (e.g., Bartel et al. 1982); XTE J1810-197 displays a much greater variety. However, the root cause of mode changing is not well understood, and as we have established that XTE J1810-197 profiles can change suddenly (Fig. 5), some link may exist between these two phenomena. Other behavior also appears extraordinary, such as the broader pulse components typically observed after MJD 53940 even as the phase separation between components remains fixed (Fig. 1 and $\S 2.2$ ) and the polarimetric properties remain largely unchanged (Camilo et al. 2007).

Compared to such extreme radio variability, the X-ray spectrum and pulse profiles of XTE J1810-197 change slowly as the flux decays (e.g., Gotthelf \& Halpern 2007), on a much longer timescale $(\sim 1 \mathrm{yr})$ than the fluctuations in torque reported here. The alignment of the peaks of the radio and X-ray pulses suggests that the footpoints of the active magnetic field lines on which radio emission is generated are also the locations of concentrated crustal heating that is responsible for the enhanced $\mathrm{X}$-ray emission, at least at the higher energies that apparently come from a relatively small area (see Gotthelf \& Halpern 2007). However, even if the energetic particle bombardment that heats the surface hot spot fluctuates on timescales of less than 1 day, the absence of correlated X-ray variability on similar timescales indicates that most of the X-ray luminosity originated in deeper crustal heating at the time of the X-ray turn on, or from more gradual decay of the magnetic field (Eichler \& Cheng 1989).

Among persistent AXPs it is not altogether clear what is the relationship between observed radiative properties (such as pulse shapes and fluxes) and rotational evolution (such as variations in torque). Pulse profiles can change following glitches (e.g., Kaspi et al. 2003), but otherwise appear to be fairly stable (Gavriil \& Kaspi 2002). Quite apart from the phenomenon of X-ray bursts (for bursts in XTE J1810-197, see Woods et al. 2005), some AXPs have shown substantial variability in X-ray flux (e.g., Gavriil \& Kaspi 2004). Nevertheless, with the possible exception of 1E 1048.1-5937 (Gavriil \& Kaspi 2004), there has been no reported correlation between X-ray flux and spin-down rate. For XTE J1810-197, on the other hand, there is an observed correlation between radio flux density and torque over a period of 9 months (Fig. 4), although we know neither whether 
this correlation is causal nor whether it holds over longer periods of time.

With observations of XTE J1810-197, we now find ourselves in the curious position of relying on energetically insignificant radio pulsations exhibiting a remarkably diverse phenomenology to illuminate rather more energetic events on the magnetar. Attempting to understand key aspects of the radio observations may lead to a deeper understanding of both magnetars and radio pulsar emission.

We are grateful to John Sarkissian for help with Parkes observations, Eric Gerard for useful discussions concerning Nançay flux calibration, and David Nice for wisdom on time systems as used in TEMPO. The Nançay radio telescope is part of the
Paris Observatory, associated with the Centre National de la Recherche Scientifique (CNRS), and partially supported by the Region Centre in France. The National Radio Astronomy Observatory is a facility of the National Science Foundation, operated under cooperative agreement by Associated Universities, Inc. The Parkes Observatory is part of the Australia Telescope, which is funded by the Commonwealth of Australia for operation as a National Facility managed by CSIRO. F. C. thanks the NSF for support through grant AST-05-07376. E. V. G. acknowledges support for this work provided by the National Aeronautics and Space Administration through Chandra Award GO6-7044X issued by the Chandra X-ray Observatory Center, which is operated by the Smithsonian Astrophysical Observatory for and on behalf of NASA under contract NAS8-03060, and by NASA ADP grant NNG05GC43G.
Arzoumanian, Z., Nice, D. J., Taylor, J. H., \& Thorsett, S. E. 1994, ApJ, 422, 671

Backer, D. C., Dexter, M. R., Zepka, A., D., N., Wertheimer, D. J., Ray, P. S., \& Foster, R. S. 1997, PASP, 109, 61

Bartel, N., Morris, D., Sieber, W., \& Hankins, T. H. 1982, ApJ, 258, 776

Beloborodov, A. M., \& Thompson, C. 2007, ApJ, 657, 967

Burgay, M., Rea, N., Israel, G. L., Possenti, A., Burderi, L., di Salvo, T., D'Amico, N., \& Stella, L. 2006, MNRAS, 372, 410

Camilo, F., Ransom, S. M., Halpern, J. P., Reynolds, J., Helfand, D. J., Zimmerman, N., \& Sarkissian, J. 2006, Nature, 442, 892

Camilo, F., Reynolds, J., Johnston, S., Halpern, J. P., Ransom, S. M., \& van Straten, W. 2007, ApJ, 659, L37

Cognard, I., \& Theureau, G. 2006, in On the Present and Future of Pulsar Astronomy, 26th meeting of the IAU (Cambridge: Cambridge Univ. Press), 2, 36

Duncan, R. C. 2001, in AIP Conf. Proc. 556, ed. H.-Y. Chang, et al. (New York: AIP), 228

Eichler, D., \& Cheng, A. F. 1989, ApJ, 336, 360

Gavriil, F. P., \& Kaspi, V. M. 2002, ApJ, 567, 1067 2004, ApJ, 609, L67

Goldreich, P., \& Julian, W. H. 1969, ApJ, 157, 869

Gotthelf, E. V., Gavriil, F. P., Kaspi, V. M., Vasisht, G., \& Chakrabarty, D. 2002, ApJ, 564, L31

Gotthelf, E. V., \& Halpern, J. P. 2005, ApJ, 632, 1075 2007, Ap\&SS, 308, 79
Halpern, J. P., \& Gotthelf, E. V. 2005, ApJ, 618, 874

Halpern, J. P., Gotthelf, E. V., Becker, R. H., Helfand, D. J., \& White, R. L. 2005, ApJ, 632, L29

Helfand, D. J., Chatterjee, S., Brisken, W. F., Camilo, F., Reynolds, J., van Kerkwijk, M. H., Halpern, J. P., \& Ransom, S. M. 2007, ApJ, 662, 1198

Ibrahim, A. I., et al. 2004, ApJ, 609, L21

Kaplan, D. L., Escoffier, R. P., Lacasse, R. J., O’Neil, K., Ford, J. M., Ransom, S. M., Anderson, S. B., Cordes, J. M., Lazio, T. J. W., \& Kulkarni, S. R. 2005, PASP, 117, 643

Kaspi, V. M. 2007, Ap\&SS, 308, 1

Kaspi, V. M., \& Gavriil, F. P. 2003, ApJ, 596, L71

Kaspi, V. M., Gavriil, F. P., Chakrabarty, D., Lackey, J. R., \& Muno, M. P. 2001, ApJ, 558, 253

Kaspi, V. M., Gavriil, F. P., Woods, P. M., Jensen, J. B., Roberts, M. S. E., \& Chakrabarty, D. 2003, ApJ, 588, L93

Kramer, M., Lyne, A. G., O’Brien, J. T., Jordan, C. A., \& Lorimer, D. R. 2006, Science, 312, 549

Thompson, C., Duncan, R. C., Woods, P. M., Kouveliotou, C., Finger, M. H., \& van Paradijs, J. 2000, ApJ, 543, 340

Thompson, C., Lyutikov, M., \& Kulkarni, S. R. 2002, ApJ, 574, 332

Woods, P. M., Kouveliotou, C., Göğüs, E., Finger, M. H., Swank, J., Markwardt, C. B., Hurley, K., \& van der Klis, M. 2002, ApJ, 576, 381

Woods, P. M., \& Thompson, C. 2006, in Compact Stellar X-ray Sources, ed.

W. H. G. Lewin \& M. van der Klis (Cambridge: Cambridge Univ. Press), 547 Woods, P. M., et al. 2005, ApJ, 629, 985 